\title{
HIGHEST WEIGHT IRREDUCIBLE REPRESENTATIONS OF RANK 2 QUANTUM TORI
}

\author{
S. Eswara RaO And K. ZhaO
}

\begin{abstract}
For any nonzero $q \in C$ (the complex numbers), the rank 2 quantum torus $C_{q}$ is the skew Laurent polynomial algebra $C\left[t_{1}^{ \pm 1}, t_{2}^{ \pm 1}\right]$ with defining relations: $t_{2} t_{1}=q t_{1} t_{2}$ and $t_{i} t_{i}^{-1}=t_{i}^{-1} t_{i}=1$. Here we consider $C_{q}$ as the naturally associated Lie algebra. We add the one dimensional center $C c_{1}$ and the outer derivation $d_{1}$ to $C_{q}$ to get the extended torus Lie algebra $\widetilde{C}_{q}$ (and $\widehat{C}_{q}$, in a different manner), where we assume $q$ is a primitive $m$-th root of unity for $\widehat{C}_{q}$. Before this paper, there appeared highest weight representations for $\widetilde{C}_{q}$ and $\widehat{C}_{q}$ with only positive integral levels. In this paper, we define the highest weight irreducible $\left(\mathbb{Z}\right.$-graded) module $V(\phi)$ over $\widetilde{C}_{q}$ and $\widehat{C}_{q}$ for any linear map $\phi: C\left[t_{2}^{ \pm 1}\right]+C c_{1}+C d_{1} \rightarrow C$, thus the central charge (level) can be any complex numbers. We obtain the necessary and sufficient conditions for $V(\phi)$ to have finite dimensional weight spaces, thus obtaining a lot of new irreducible weight representations for these Lie algebras. The corresponding irreducible $\mathbb{Z} \times \mathbb{Z}$-graded modules with finite dimensional weight spaces over $\widetilde{C}_{q}$ are also constructed.
\end{abstract}

\section{Introduction}

In the representation theory of infinite dimensional Lie algebras, one of the main tasks is the construction of the "good" modules. Recently there has been substantial activity in developing representation theory for higher rank infinite dimensional Lie algebras, in particular toroidal Lie algebras, and quantum torus algebras (see [1], [5], [6], [7], [8], [9], [10]).

Unlike rank one algebras (affine and Virasoro), the higher rank infinite dimensional Lie algebras do not possess a triangular decomposition, which makes the standard construction of the highest weight modules inapplicable. Nonetheless, there have been found several explicit realizations of representations for these algebras using the vertex operator approach (see the above mentioned papers).

In the vertex constructions the highest weight is replaced with a module for the subalgebra of degree zero (the subalgebra is infinite dimensional). Let us describe in brief these representations from the perspective of the highest weight modules.

Let $G$ be a complex $\mathbb{Z}$-graded Lie algebra and let $G=G^{-} \oplus G^{(0)} \oplus G^{+}$be a decomposition of $G$ relative to the $\mathbb{Z}$-grading, where $\mathbb{Z}$ is the ring of integers.

Received June 1, 2003.

2000 Mathematics Subject Classification. 17B20, 17B65, 17B67, 17B68, 16A40, 16A68.

This work is supported by the NSF of China (Grant 10371120). 
The subalgebra $G^{(0)}$ is an infinite-dimensional Lie algebra, not necessarily commutative. We take some natural module $V$ for $G^{(0)}$. Parallel to the construction of a highest weight module, we let $G^{+}$act on $V$ trivially, and introduce the induced module

$$
\tilde{M}(V)=\operatorname{Ind}_{G^{(0)}+G^{+}}^{G} V \simeq U\left(G^{-}\right) \otimes_{C} V,
$$

where $C$ is the field of complex numbers. Then $\tilde{M}(V)$ is $\mathbb{Z}$-graded.

The difficulty here is that $\tilde{M}(V)$ will have infinite-dimensional homogeneous components (and thus will not have a character formula). Nonetheless the explicit vertex operator constructions show that, in some cases, $\tilde{M}(V)$ indeed has an irreducible quotient with finite-dimensional homogeneous components. This situation has been clarified in [6], where it was proved that $\tilde{M}(V)$ has a graded factor-module $M(V)$ with finite-dimensional components for some $V$ over some quantum tori $C_{q}$ defined below in (1.2) and (1.4). Now let us first recall the definition for $C_{q}$.

Let $q \in C$ be nonzero. The rank 2 q-quantum torus $C_{q}$ which (and higher rank also) was studied in [11] is the unital associative algebra over $C$ generated by $t_{1}^{ \pm 1}, t_{2}^{ \pm 1}$ and subject to the defining relations

$$
t_{2} t_{1}=q t_{1} t_{2}, t_{i} t_{i}^{-1}=t_{i}^{-1} t_{i}=1 \text {. }
$$

In this paper we always consider $C_{q}$ as the associated Lie algebra. The reason we consider only rank 2 quantum torus Lie algebras is the following. In many references like [2], [8], [9], [10] (but not [6]), higher rank quantum torus Lie algebras are studied but with the assumption that all the variables except $t_{1}$ are commutative. Algebras under this assumption essentially have the same properties which assure that they have the same type representations.

For any $a \in \mathbb{Z}^{2}$, we always write $a=\left(a_{1}, a_{2}\right)$, and denote $t^{a}=t_{1}^{a_{1}} t_{2}^{a_{2}}$. For any $a, b \in \mathbb{Z}^{2}$, we define $\sigma(a, b)$ and $f(a, b)$ by

$$
t^{a} t^{b}=\sigma(a, b) t^{a+b}, t^{a} t^{b}=f(a, b) t^{b} t^{a} .
$$

Then

$$
\begin{gathered}
\sigma(a, b)=q^{a_{2} b_{1}}, f(a, b)=q^{a_{2} b_{1}-a_{1} b_{2}}, \text { and } \\
f(a, b)=\sigma(a, b) \sigma(b, a)^{-1} .
\end{gathered}
$$

For properties of $C_{q}$, please refer to [2] or [12]. Define $\operatorname{rad} f=\left\{a \in \mathbb{Z}^{2} \mid f\left(a, \mathbb{Z}^{2}\right)\right.$ $=1\}$ and

$$
\delta_{\alpha, \operatorname{rad} f}= \begin{cases}1, & \text { if } \alpha \in \operatorname{rad} f \\ 0, & \text { otherwise. }\end{cases}
$$

Let $\widetilde{C}_{q}=C_{q} \oplus C c_{1} \oplus C d_{1}$ be the extension of the Lie algebra $C_{q}$ with defining relations

$$
\begin{gathered}
{\left[t^{\alpha}, t^{\beta}\right]=t^{\alpha} t^{\beta}-t^{\beta} t^{\alpha}+\delta_{\alpha+\beta, 0} q^{-\alpha_{1} \alpha_{2}} \alpha_{1} c_{1}, \quad \forall \alpha, \beta \in \mathbb{Z}^{2},} \\
{\left[c_{1}, t^{\alpha}\right]=0, \quad\left[d_{1}, t^{\alpha}+C c_{1}\right]=\alpha_{1} t^{\alpha}, \quad \forall \alpha \in \mathbb{Z}^{2},}
\end{gathered}
$$

where $\delta_{\alpha+\beta, 0}$ is the Kroneker delta. 
If $q$ is a primitive $m$-th root of unity (we assume that $m>1$ otherwise $C_{q}$ is commutative, a case which does not concern us), we can similarly add the center $c_{1}$ and the outer derivation $d_{1}$ to $C_{q}$ to get the extended torus Lie algebras $\widehat{C}_{q}$ with defining relations:

$$
\begin{gathered}
{\left[t^{\alpha}, t^{\beta}\right]=t^{\alpha} t^{\beta}-t^{\beta} t^{\alpha}+\delta_{\alpha_{1}+\beta_{1}, 0} \delta_{\alpha+\beta, \operatorname{rad} f} q^{-\alpha_{1} \alpha_{2}} \alpha_{1} c_{1}, \quad \forall \alpha, \beta \in \mathbb{Z}^{2},} \\
{\left[c_{1}, t^{\alpha}\right]=0,\left[d_{1}, t^{\alpha}+C c_{1}\right]=\alpha_{1} t^{\alpha} .}
\end{gathered}
$$

It is clear that $\widetilde{C}_{q}$ and $\widehat{C}_{q}$ have a $\mathbb{Z}$-gradation with respect to $C d_{1}$ :

$$
\widetilde{C}_{q}=\widehat{C}_{q}=\oplus_{k \in \mathbb{Z}} L_{k}
$$

where $L_{k}=\oplus_{p \in \mathbb{Z}} C t_{1}^{k} t_{2}^{p} \oplus \delta_{k, 0}\left(C c_{1}+C d_{1}\right)$. For a $\mathbb{Z}$-graded module $V=\oplus_{i \in \mathbb{Z}} V_{k}$ over $\widetilde{C}_{q}$ or $\widehat{C}_{q}$, if it has finite dimensional homogeneous subspaces, i.e., $\operatorname{dim} V_{k}<$ $\infty$ for all $k \in \mathbb{Z}$, its character is defined as

$$
\operatorname{ch} V=\sum_{k \in \mathbb{Z}}\left(\operatorname{dim} V_{k}\right) z^{-k}
$$

Before this paper, there appeared only highest weight representations with finite dimensional weight spaces for $\widetilde{C}_{q}$ and $\widehat{C}_{q}$ with level one or other positive integral levels (see [2], [8], [9], [5], [6]). In this paper, we define the highest weight irreducible $\left(\mathbb{Z}\right.$-graded) module $V(\phi)$ over $\widetilde{C}_{q}$ and $\widehat{C}_{q}$ for any linear map $\phi: C\left[t_{2}^{ \pm 1}\right]+C c_{1}+C d_{1} \rightarrow C$, thus the central charge (level) can be any complex numbers. We obtain the necessary and sufficient conditions for $V(\phi)$ to have finite dimensional weight spaces (Theorems $2.2,2.4,2.5$ ), thus obtaining a lot of new irreducible weight representations. The corresponding irreducible $\mathbb{Z} \times \mathbb{Z}$ graded modules with finite dimensional homogeneous subspaces over $\widetilde{C}_{q}$ are given in Section 3.

\section{Highest weight representations for $\widetilde{C}_{q}$ and $\widehat{C}_{q}$}

In this section we denote $L=\widetilde{C}_{q}$ (or $\widehat{C}_{q}$ ). With respect to the $\mathbb{Z}$-gradation (1.6), let $L_{+}=\oplus_{i \in \mathbb{Z}_{+}} L_{i}, L_{-}=\oplus_{i<0} L_{i}$.

Now we define highest weight modules over $L$. For any linear map

$$
\phi: C\left[t_{2}^{ \pm 1}\right]+C c_{1}+C d_{1} \rightarrow C
$$

with $\phi\left(d_{1}\right)=0$ (this is only for convenience since these values do not affect the module structure), we define the 1 -dimensional $\left(L_{0}+L_{+}\right)$-module $C v_{0}$ via

$$
L_{i} v_{0}=0, \text { if } i>0 ; x v_{0}=\phi(x) v_{0}, \forall x \in L_{0} .
$$

Then we have the induced $L$-module

$$
\bar{V}(\phi)=\operatorname{Ind}_{L_{0}+L_{+}}^{L} C v_{0}=U(L) \otimes_{U\left(L_{+}+L_{0}\right)} C v_{0},
$$

where $U(L)$ is the universal enveloping algebra of the Lie algebra $L$. It is clear that, $d_{1}$ acts diagonally on $\bar{V}(\phi)$, and $\bar{V}(\phi) \simeq U\left(L_{-}\right)$as vector spaces. Since 
the 0-eigenspace of $d_{1}$ is 1-dimensional, the module $\bar{V}(\phi)$ has a unique maximal proper submodule $J$. Then we obtain the irreducible module

$$
V(\phi)=\frac{\bar{V}(\phi)}{J}
$$

It is clear that $V(\phi)$ is uniquely determined by the linear function $\phi$, and $V(\phi)=$ $\oplus_{i \in \mathbb{Z}_{+}} V_{-i}$ where

$$
V_{i}=\left\{v \in V(\phi) \mid d_{1} v=i v\right\} .
$$

Generally, not all weight spaces $V_{i}$ of $V(\phi)$ are finite-dimensional.

Theorem 2.1. (a) The module $V(\phi)$ over $\widetilde{C}_{q}$ has finite dimensional weight spaces if and only if there exists a nonzero polynomial $P\left(t_{2}\right)=\sum_{i=0}^{n} a_{i} t_{2}^{i} \in C\left[t_{2}\right]$ with $a_{0} a_{n} \neq 0$ such that

$$
\phi\left(t_{2}^{k} P\left(t_{2}\right)-q^{k} t_{2}^{k} P\left(q t_{2}\right)+a_{-k} c_{1}\right)=0, \quad \forall k \in \mathbb{Z},
$$

and further $\phi\left(c_{1}\right)=0$ if $q$ is not generic (i.e., $q$ is a root of unity), where $a_{k}=0$ if $k \notin\{0,1, \ldots, n\}$.

(b) Suppose $q$ is a primitive $m$-th root of unity with $m>1$. The module $V(\phi)$ over $\widehat{C}_{q}$ has finite dimensional weight spaces if and only if there exists a nonzero polynomial $P\left(t_{2}\right)=\sum_{i=0}^{n} a_{i} t_{2}^{i} \in C\left[t_{2}\right]$ with $a_{0} a_{n} \neq 0$ such that

$$
\phi\left(t_{2}^{k} P\left(t_{2}\right)-q^{k} t_{2}^{k} P\left(q t_{2}\right)+\sum_{i \equiv-k \bmod m} a_{i} c_{1}\right)=0, \quad \forall k \in \mathbb{Z},
$$

where $a_{k}=0$ if $k \notin\{0,1, \cdots, n\}$.

Proof. (a) " $\Rightarrow$ ". Since $\operatorname{dim} V_{-1}<\infty$, there exist an $s \in \mathbb{Z}$ and a nonzero polynomial $P\left(t_{2}\right)=\sum_{i=0}^{n} a_{i} t_{2}^{i} \in C\left[t_{2}\right]$ with $a_{0} a_{n} \neq 0$ such that

$$
\left(t_{1}^{-1} t_{2}^{s} P\left(t_{2}\right)\right) \cdot v_{0}=0 \text {. }
$$

Applying $t_{1} t_{2}^{k}$ for any $k \in \mathbb{Z}$ to the above equation, we obtain that

$$
\begin{gathered}
0=\left(t_{1} t_{2}^{k}\right) \cdot\left(t_{1}^{-1} t_{2}^{s} P\left(t_{2}\right)\right) \cdot v_{0}=q^{-k}\left(t_{2}^{k+s} P\left(t_{2}\right)-q^{k+s} t_{2}^{k+s} P\left(q t_{2}\right)+a_{-k-s} c_{1}\right) v_{0} \\
=q^{-k} \phi\left(t_{2}^{k+s} P\left(t_{2}\right)-q^{k+s} t_{2}^{k+s} P\left(q t_{2}\right)+a_{-k-s} c_{1}\right) v_{0}
\end{gathered}
$$

to give

$$
\phi\left(t_{2}^{k+s} P\left(t_{2}\right)-q^{k+s} t_{2}^{k+s} P\left(q t_{2}\right)+a_{-k-s} c_{1}\right)=0 .
$$

If $q$ is a primitive $m$-th root of 1 where $m \geq 1$, and $\phi\left(c_{1}\right) \neq 0$, by applying $t_{1}^{m} t_{2}^{p}$ to $\sum_{i} b_{i}\left(t_{1}^{-m} t_{2}^{i}\right) v_{0}=0$ we deduce that $b_{i}=0$, so $\left\{\left(t_{1}^{-m} t_{2}^{i}\right) v_{0} \mid i \in \mathbb{Z}\right\} \subset V_{-m}$ is a linearly independent set, contrary to the assumption. So $\phi\left(c_{1}\right)=0$ if $q$ is a primitive $m$-th root of 1 . Thus this direction follows.

"६". For $V_{0}$, we know that

$$
\left(t_{2}^{k} P\left(t_{2}\right)-q^{k} t_{2}^{k} P\left(q t_{2}\right)+a_{-k} c_{1}\right) \cdot v_{0}=0, \quad \forall k \in \mathbb{Z} .
$$


Since for any $k, l \in \mathbb{Z}$,

$$
\begin{gathered}
\left(t_{1} t_{2}^{k}\right) \cdot\left(t_{1}^{-1} t_{2}^{l} P\left(t_{2}\right)\right) \cdot v_{0}=q^{-k}\left(t_{2}^{k+l} P\left(t_{2}\right)-q^{k+l} t_{2}^{k+l} P\left(q t_{2}\right)+a_{-k-l} c_{1}\right) v_{0} \\
=q^{-k} \phi\left(t_{2}^{k+l} P\left(t_{2}\right)-q^{k+l} t_{2}^{k+l} P\left(q t_{2}\right)+a_{-k-l} c_{1}\right) v_{0}=0
\end{gathered}
$$

from the irreducibility of $V(\phi)$ we see that

$$
\left(t_{1}^{-1} t_{2}^{l} P\left(t_{2}\right)\right) \cdot v_{0}=0, \quad \forall l \in \mathbb{Z} .
$$

Note that $L_{-}$is generated by $L_{-1}$ and $t^{\alpha} \in L_{-}$for $\alpha \in \operatorname{rad} f$, and $L_{+}$is generated by $L_{1}$ and $t^{\alpha} \in L_{+}$for $\alpha \in \operatorname{rad} f$. For any $t^{\alpha} \in L_{-}$with $\alpha \in \operatorname{rad} f$ it is clear that $L_{+} t^{\alpha} v_{0}=0$, thus we deduce that $t^{\alpha} V=0$ for any $\alpha \in \operatorname{rad} f$. Further

$$
L_{-1} V_{-i}=V_{-i-1}, \quad \forall i \in \mathbb{Z}_{+},
$$

and, if $v \in V_{-i}$ where $i>0$, satisfies $L_{1} v=0$ then $v=0$.

Next, by induction on $s$ we show

Claim 1. For any $i: 0 \leq i \leq s$ where $s \in \mathbb{Z}_{+}$, we have nonzero finite sum $P_{i}\left(t_{2}\right)=\sum_{j \in \mathbb{Z}} a_{j}^{(i)} t_{2}^{j} \in C\left[t_{2}\right]$ such that

$$
\begin{gathered}
\left(t_{2}^{k} P_{i}\left(t_{2}\right)-q^{k} t_{2}^{k} P_{i}\left(q t_{2}\right)+a_{-k}^{(i)} c_{1}\right) V_{-i}=0, \quad \forall k \in \mathbb{Z}, \\
\left(t_{1}^{-1} t_{2}^{k} P_{i}\left(t_{2}\right)\right) V_{-i}=0, \quad \forall k \in \mathbb{Z} .
\end{gathered}
$$

Formulae (2.6) and (2.7) ensure the claim for $s=0$ with $P_{0}=P$. Suppose the claim holds for $s$. Now let us consider the claim for $s+1$.

The first formula in the Claim is equivalent to

$$
\left(Q\left(t_{2}\right)-Q\left(q t_{2}\right)+a_{Q} c_{1}\right) \cdot V_{-i}=0,
$$

for any $Q\left(t_{2}\right) \in C\left[t_{2}^{ \pm 1}\right]$ with $P_{i} \mid Q$, where $a_{Q}$ is the constant term of $Q$.

Let $P_{s+1}\left(t_{2}\right)=P_{s}\left(q t_{2}\right) P_{s}\left(t_{2}\right) P_{s}\left(q^{-1} t_{2}\right)=\sum_{j \in \mathbb{Z}} a_{j}^{(s+1)} t_{2}^{j}$. For any $k, l \in$ $\mathbb{Z}$, noticing that $P_{s}\left(t_{2}\right)\left|P_{s+1}\left(t_{2}\right), P_{s}\left(t_{2}\right)\right| P_{s+1}\left(q t_{2}\right)$ and $P_{s}\left(t_{2}\right) \mid P_{s+1}\left(q^{-1} t_{2}\right)$, by induction we have

$$
\begin{gathered}
\left(t_{2}^{l} P_{s+1}\left(t_{2}\right)-q^{l} t_{2}^{l} P_{s+1}\left(q t_{2}\right)+a_{-l}^{(s+1)} c_{1}\right) \cdot\left(t_{1}^{-1} t_{2}^{k} V_{-s}\right) \\
=\left(t_{1}^{-1} t_{2}^{k}\right)\left(t_{2}^{l} P_{s+1}\left(t_{2}\right)-q^{l} t_{2}^{l} P_{s+1}\left(q t_{2}\right)+a_{-l}^{(s+1)} c_{1}\right) \cdot V_{-s} \\
+\left[t_{2}^{l} P_{s+1}\left(t_{2}\right)-q^{l} t_{2}^{l} P_{s+1}\left(q t_{2}\right)+a_{-l}^{(s+1)} c_{1}, t_{1}^{-1} t_{2}^{k}\right] V_{-s} \\
=\left(t_{1}^{-1} t_{2}^{k+l}\left(q^{-l} P_{s+1}\left(q^{-1} t_{2}\right)-2 P_{s+1}\left(t_{2}\right)+q^{l} P_{s+1}\left(q t_{2}\right)\right)\right) V_{-s}=0 .
\end{gathered}
$$

This proves the first formula in Claim 1 for $i=s+1$.

Using this newly established formula, for any $k, l, r \in \mathbb{Z}$, noticing that $\left(t_{1}^{-1} t_{2}^{l} P_{s+1}\left(t_{2}\right)\right) \cdot V_{-s}=0$, we deduce that

$$
\begin{gathered}
\left(t_{1} t_{2}^{r}\right) \cdot\left(t_{1}^{-1} t_{2}^{l} P_{s+1}\left(t_{2}\right)\right) \cdot\left(t_{1}^{-1} t_{2}^{k} V_{-s}\right) \\
=\left[t_{1} t_{2}^{r}, t_{1}^{-1} t_{2}^{l} P_{s+1}\left(t_{2}\right)\right] \cdot\left(t_{1}^{-1} t_{2}^{k} V_{-s}\right)+\left(t_{1}^{-1} t_{2}^{l} P_{s+1}\left(t_{2}\right)\right) \cdot\left(t_{1} t_{2}^{r}\right) \cdot\left(t_{1}^{-1} t_{2}^{k} V_{-s}\right)
\end{gathered}
$$




$$
\begin{gathered}
=\left[t_{1} t_{2}^{r}, t_{1}^{-1} t_{2}^{l} P_{s+1}\left(t_{2}\right)\right] \cdot\left(t_{1}^{-1} t_{2}^{k} V_{-s}\right) \\
=q^{-r}\left(t_{2}^{r+l} P_{s+1}\left(t_{2}\right)-q^{r+l} t_{2}^{r+l} P_{s+1}\left(q t_{2}\right)+a_{-r-l}^{(s+1)} c_{1}\right) \cdot\left(t_{1}^{-1} t_{2}^{k} V_{-s}\right)=0,
\end{gathered}
$$

which implies that $\left(t_{1}^{-1} t_{2}^{l} P_{s+1}\left(t_{2}\right)\right) \cdot\left(t_{1}^{-1} t_{2}^{k} V_{-s}\right)=0$ for all $k, l \in \mathbb{Z}$. Thus $\left(t_{1}^{-1} t_{2}^{l} P_{s+1}\left(t_{2}\right)\right) \cdot V_{-s-1}=0$ for all $l \in \mathbb{Z}$. This proves the second formula in Claim 1 for $i=s+1$. By inductive principle, therefore our Claim follows.

From the second formula of Claim 1, we see that

$$
\operatorname{dim} V_{-s-1} \leq \operatorname{deg} P_{s+1} \cdot \operatorname{dim} V_{-s}, \quad \forall s \in \mathbb{Z}_{+} .
$$

Thus Part (a) holds.

(b) " $\Rightarrow$ ". This is similar to the proof of the corresponding part of (a). We omit the details.

" $\Leftarrow$ ". If $\phi\left(c_{1}\right)=0$, this is (a). Next suppose $\phi\left(c_{1}\right) \neq 0$.

Let $H=\oplus_{\alpha \in \operatorname{rad} f} C t^{\alpha} \oplus C c_{1} \oplus C d_{1}, L^{\prime}=[L, L]$ and $K=\oplus_{i \in \mathbb{Z}} C t_{1}^{i m} \oplus C c_{1} \oplus$ $C d_{1}$. Then $H, K$ and $L^{\prime}$ are Lie subalgebras of $L, L=H+L^{\prime}$ with $\left[H, L^{\prime}\right]=0$, and $K$ is the standard Heisenberg algebra with the degree operator $d_{1}$.

Let $W=U(H) v_{0}$. We see that $V=U\left(L^{\prime}\right) U(H) v_{0}$,

$$
t^{\alpha} U(H) v_{0}=0, \quad \forall t^{\alpha} \in L_{+} \cap L^{\prime},
$$

Claim 2. $\left.t_{1}^{m i} t_{2}^{m j}\right|_{W}=\left.t_{1}^{m i}\right|_{W}$ for all $i, j \in \mathbb{Z}$.

We show this claim by induction on $W_{-m k}$ for $k \in \mathbb{Z}_{+}$. It is easy to verify that

$$
t^{\alpha}\left(\left(t_{1}^{m i} t_{2}^{m j}-t_{1}^{m i}\right) W_{0}\right)=0, \quad \forall t^{\alpha} \in L_{+} .
$$

Then $\left(t_{1}^{m i} t_{2}^{m j}-t_{1}^{m i}\right) W_{0}=0$, i.e., $\left.t_{1}^{m i} t_{2}^{m j}\right|_{W_{0}}=\left.t_{1}^{m i}\right|_{W_{0}}$.

Suppose $\left.t_{1}^{m i} t_{2}^{m j}\right|_{-m k}=\left.t_{1}^{m i}\right|_{W_{-k m}}$ for all $k \leq k_{0}$. For any $w \in W_{-(k+1) m}$, by computing

$$
\left.\left.t^{\alpha}\left(\left(t_{1}^{m i} t_{2}^{m j}\right)-t_{1}^{m i}\right) w\right)=\left(t_{1}^{m i} t_{2}^{m j}\right)-t_{1}^{m i}\right)\left(t^{\alpha}(w)\right)=0, \quad \forall t^{\alpha} \in L_{+},
$$

we see that $\left.t_{1}^{m i} t_{2}^{m j}\right|_{W_{k+1}}=\left.t_{1}^{m i}\right|_{W_{k+1}}$. Hence Claim 2 follows.

From Claim 2 we know that $W=U(K) v_{0}, W$ is an irreducible $K$-module, and

$$
\operatorname{dim} W_{m k}<\infty, \quad \forall k \in \mathbb{Z} .
$$

Let $L_{-}^{\prime}=L^{\prime} \cap L_{-}$and $L_{+}^{\prime}=L^{\prime} \cap L_{+}$. It is clear that

$$
V_{-k}=\sum_{j \geq 0, i+m j=k} U\left(L_{-}^{\prime}\right)_{-i} W_{-m j},
$$

where $U\left(L_{-}^{\prime}\right)_{-i}=\left\{u \in U\left(L_{-}^{\prime}\right) \mid\left[d_{1}, u\right]=-i u\right\}$, and the right hand side of $(2.11)$ is a finite sum.

Since $L_{-}^{\prime}$ is generated by $L_{-1}^{\prime}$, and $L_{+}^{\prime}$ is generated by $L_{1}^{\prime}$, we deduce that

$$
L_{-1}^{\prime} W_{-i}=W_{-i-1}, \quad \forall i \in \mathbb{Z}_{+},
$$

and, if $v \in W_{-i}$ satisfies $L_{1}^{\prime} v=0$, where $i>0$, then $v=0$. 
From (2.10) and (2.11), it suffices to show that, for any homogeneous $u \in W$, the $L^{\prime}$-module $W^{\prime}=U\left(L^{\prime}\right) u$ has finite dimensional weight spaces. Since $V$ is an irreducible $L$-module, $W^{\prime}$ is an irreducible $L^{\prime}$-module.

We simply write $W_{-i}^{\prime}=U\left(L^{\prime}\right)_{-i} u$. For $W_{0}^{\prime}$, from (2.5) we know that

$$
\left(t_{2}^{k} P\left(t_{2}\right)-q^{k} t_{2}^{k} P\left(q t_{2}\right)+\sum_{i \equiv-k \bmod m} a_{i} c_{1}\right) \cdot u=0, \quad \forall k \in \mathbb{Z} .
$$

Since for any $k, l \in \mathbb{Z}$,

$$
\begin{gathered}
\left(t_{1} t_{2}^{k}\right) \cdot\left(t_{1}^{-1} t_{2}^{l} P\left(t_{2}\right)\right) \cdot u=q^{-k}\left(t_{2}^{k+l} P\left(t_{2}\right)-q^{k+l} t_{2}^{k+l} P\left(q t_{2}\right)+\sum_{i \equiv-k-l \bmod m} a_{i} c_{1}\right) u \\
=q^{-k} \phi\left(t_{2}^{k+l} P\left(t_{2}\right)-q^{k+l} t_{2}^{k+l} P\left(q t_{2}\right)+\sum_{i \equiv-k-l \bmod m} a_{i} c_{1}\right) u=0
\end{gathered}
$$

from the irreducibility of $W^{\prime}$ we see that

$$
\left(t_{1}^{-1} t_{2}^{l} P\left(t_{2}\right)\right) \cdot u=0, \quad \forall l \in \mathbb{Z} .
$$

Next, using the same technique as in the proof of Claim 1 by induction on $s$ we show

Claim 3. For any $i: 0 \leq i \leq s$ where $s \in \mathbb{Z}_{+}$, we have nonzero $P_{i}\left(t_{2}\right)=$ $\sum_{j \in \mathbb{Z}} a_{j}^{(i)} t_{2}^{j} \in C\left[t_{2}\right]$ such that

$$
\begin{gathered}
\left(t_{2}^{k} P_{i}\left(t_{2}\right)-q^{k} t_{2}^{k} P_{i}\left(q t_{2}\right)+\sum_{i \equiv-k \bmod m} a_{-k}^{(i)} c_{1}\right) W_{-i}=0, \quad \forall k \in \mathbb{Z}, \\
\left(t_{1}^{-1} t_{2}^{k} P_{i}\left(t_{2}\right)\right) W_{-i}=0, \quad \forall k \in \mathbb{Z} .
\end{gathered}
$$

Formulae (2.12) and (2.13) ensure the claim for $s=0$ with $P_{0}=P$. Suppose the claim holds for $s$. Now let us consider the claim for $s+1$.

The first formula in Claim 3 is equivalent to

$$
\left(Q\left(t_{2}\right)-Q\left(q t_{2}\right)+\sum_{i \equiv 0 \bmod m} b_{i} c_{1}\right) \cdot W_{-i}=0
$$

for any $Q\left(t_{2}\right)=\sum b_{i} t_{2}^{i}$ with $P_{i} \mid Q$.

Let $P_{s+1}\left(t_{2}\right)=P_{s}\left(q t_{2}\right) P_{s}\left(t_{2}\right) P_{s}\left(q^{-1} t_{2}\right)=\sum_{j \in \mathbb{Z}} a_{j}^{(s+1)} t_{2}^{j}$. For any $k, l \in$ $\mathbb{Z}$, noticing that $P_{s}\left(t_{2}\right)\left|P_{s+1}\left(t_{2}\right), P_{s}\left(t_{2}\right)\right| P_{s+1}\left(q t_{2}\right)$ and $P_{s}\left(t_{2}\right) \mid P_{s+1}\left(q^{-1} t_{2}\right)$, by induction we have

$$
\begin{gathered}
\left(t_{2}^{l} P_{s+1}\left(t_{2}\right)-q^{l} t_{2}^{l} P_{s+1}\left(q t_{2}\right)+\sum_{i \equiv-l \bmod m} a_{i}^{(s+1)} c_{1}\right) \cdot\left(t_{1}^{-1} t_{2}^{k} W_{-s}\right) \\
=\left(t_{1}^{-1} t_{2}^{k}\right)\left(t_{2}^{l} P_{s+1}\left(t_{2}\right)-q^{l} t_{2}^{l} P_{s+1}\left(q t_{2}\right)+\sum_{i \equiv-l \bmod m} a_{i}^{(s+1)} c_{1}\right) \cdot W_{-s} \\
+\left[t_{2}^{l} P_{s+1}\left(t_{2}\right)-q^{l} t_{2}^{l} P_{s+1}\left(q t_{2}\right)+\sum_{i \equiv-l \bmod m} a_{i}^{(s+1)} c_{1}, t_{1}^{-1} t_{2}^{k}\right] W_{-s}
\end{gathered}
$$




$$
=\left(t_{1}^{-1} t_{2}^{k+l}\left(q^{-l} P_{s+1}\left(q^{-1} t_{2}\right)-2 P_{s+1}\left(t_{2}\right)+q^{l} P_{s+1}\left(q t_{2}\right)\right)\right) V_{-s}=0 .
$$

This proves the first formula in Claim 3 for $i=s+1$. Using this newly established formula, for any $k, l, r \in \mathbb{Z}$, noticing that $\left(t_{1}^{-1} t_{2}^{l} P_{s+1}\left(t_{2}\right)\right) . W_{-s}=0$, we deduce that

$$
\begin{gathered}
\left(t_{1} t_{2}^{r}\right) \cdot\left(t_{1}^{-1} t_{2}^{l} P_{s+1}\left(t_{2}\right)\right) \cdot\left(t_{1}^{-1} t_{2}^{k} W_{-s}\right) \\
=\left[t_{1} t_{2}^{r}, t_{1}^{-1} t_{2}^{l} P_{s+1}\left(t_{2}\right)\right] \cdot\left(t_{1}^{-1} t_{2}^{k} W_{-s}\right)+\left(t_{1}^{-1} t_{2}^{l} P_{s+1}\left(t_{2}\right)\right) \cdot\left(t_{1} t_{2}^{r}\right) \cdot\left(t_{1}^{-1} t_{2}^{k} W_{-s}\right) \\
=\left[t_{1} t_{2}^{r}, t_{1}^{-1} t_{2}^{l} P_{s+1}\left(t_{2}\right)\right] \cdot\left(t_{1}^{-1} t_{2}^{k} W_{-s}\right) \\
=q^{-r}\left(t_{2}^{r+l} P_{s+1}\left(t_{2}\right)-q^{r+l} t_{2}^{r+l} P_{s+1}\left(q t_{2}\right)+\sum_{i \equiv-r-l \bmod m} a_{i}^{(s+1)} c_{1}\right) \cdot\left(t_{1}^{-1} t_{2}^{k} W_{-s}\right)=0,
\end{gathered}
$$

which implies that $\left(t_{1}^{-1} t_{2}^{l} P_{s+1}\left(t_{2}\right)\right) \cdot\left(t_{1}^{-1} t_{2}^{k} W_{-s}\right)=0$ for all $k, l \in \mathbb{Z}$. Thus $\left(t_{1}^{-1} t_{2}^{l} P_{s+1}\left(t_{2}\right)\right) \cdot W_{-s-1}=0$ for all $l \in \mathbb{Z}$. This proves the second formula in Claim 3 for $i=s+1$. Therefore our Claim follows.

From the second formula of Claim 3 we see that

$$
\operatorname{dim} W_{-s-1} \leq \operatorname{deg} P_{s+1} \cdot \operatorname{dim} W_{-s}, \quad \forall s \in \mathbb{Z}_{+} .
$$

Thus Part (b) holds. Our theorem follows.

Theorem 2.2. Suppose $q$ is generic. Then the module $V(\phi)$ over $\widetilde{C}_{q}=\widehat{C}_{q}$ has finite dimensional homogeneous subspaces if and only if there exist a positive integer $r$ and $b_{10}, b_{11}, \ldots, b_{1 s_{1}}, \ldots, b_{r 0}, b_{r 1}, \ldots, b_{r s_{r}} \in C, \alpha_{1}, \ldots, \alpha_{r} \in C^{*}$ such that

$$
\begin{aligned}
\phi\left(t_{2}^{i}\right)=\frac{\left(b_{10}+b_{11} i+\ldots+b_{1 s_{1}} i^{s_{1}}\right) \alpha_{1}^{i}+\ldots+\left(b_{r 0}+b_{r 1} i+\ldots+b_{r s_{r}} i^{s_{r}}\right) \alpha_{r}^{i}}{1-q^{i}}, & \forall i \in \mathbb{Z} \backslash\{0\}, \\
\phi\left(c_{1}\right) & =b_{10}+b_{20}+\ldots+b_{r 0} .
\end{aligned}
$$

Proof. " $\Rightarrow$ ". Suppose $f_{i}=\phi\left(\left(1-q^{i}\right) t_{2}^{i}\right)$ for $i \in \mathbb{Z} \backslash\{0\}$ and $f_{0}=\phi\left(c_{1}\right)$. Then (2.4) becomes

$$
\sum_{i=0}^{n} a_{i} f_{k+i}=0, \quad \forall k \in \mathbb{Z} .
$$

Suppose $\alpha_{1}, \alpha_{2}, \ldots, \alpha_{r}$ are all distinct roots of the equation $P\left(t_{2}\right)=0$ with multiplicity $s_{1}+1, s_{2}+1, \ldots, s_{r}+1$ respectively. Then by a well-known combinatorial formula, we know that there exist $b_{10}, b_{11}, \ldots, b_{1 s_{1}}, \ldots, b_{r 0}, b_{r 1}, \ldots, b_{r s_{r}} \in C$ such that

$$
f_{i}=\left(b_{10}+b_{11} i+\ldots+b_{1 s_{1}} i^{s_{1}}\right) \alpha_{1}^{i}+\ldots+\left(b_{r 0}+b_{r 1} i+\ldots+b_{r s_{r}} i^{s_{r}}\right) \alpha_{r}^{i}, \quad \forall i \in \mathbb{Z} .
$$

Then, for all $i \in \mathbb{Z} \backslash\{0\}$,

$$
\begin{aligned}
& \begin{aligned}
\left(1-q^{i}\right) \phi\left(t_{2}^{i}\right)=\left(b_{10}+b_{11} i+\ldots+\right. & \left.+b_{1 s_{1}} i^{s_{1}}\right) \alpha_{1}^{i}+\ldots \\
& +\left(b_{r 0}+b_{r 1} i+\ldots+b_{r s_{r}} i^{s_{r}}\right) \alpha_{r}^{i}, \text { and }
\end{aligned} \\
& \phi\left(c_{1}\right)=b_{10}+b_{20}+\ldots+b_{r 0} .
\end{aligned}
$$


Thus we have the expression for $\phi\left(t_{2}^{i}\right)$ for $i \in \mathbb{Z} \backslash\{0\}$, and $\phi\left(c_{1}\right)$ in the theorem. This direction follows.

" $\Leftarrow$ ". Let $P\left(t_{2}\right)=\prod_{i=1}^{r}\left(t_{2}-\alpha_{i}\right)^{s_{i}+1}$. By using the above used combinatorial formula we can easily verify that (2.15) holds, i.e., (2.4) holds. This direction holds. This completes the proof of this theorem.

Next we suppose that $q$ is a primitive $m$-th root of unity with $m>1$. We first establish the following Lemma.

Lemma 2.3. Suppose $r>1, a_{1}, a_{2}, \ldots, a_{r}, \beta_{1}, \beta_{2}, \ldots, \beta_{r} \in C$ with $\left|\beta_{1}\right|=\left|\beta_{2}\right|=$ $\ldots=\left|\beta_{r}\right|=1$ and $\beta_{1}, \beta_{2}, \ldots, \beta_{r}$ are pair-wise distinct. If

$$
\lim _{i \in \mathbb{Z}, i \rightarrow \infty}\left(a_{1} \beta_{1}^{i}+a_{2} \beta_{2}^{i}+\ldots+a_{r} \beta_{r}^{i}\right)
$$

exists, then $a_{1}=a_{2}=\ldots=a_{r}=0$, or only one $a_{i}$ is not zero and the corresponding $\beta_{i}=1$.

Proof. Suppose only one $a_{k}$ is not zero, say $a_{1} \neq 0$. Then $\lim _{i \in \mathbb{Z}, i \rightarrow \infty} \beta_{1}^{i}=\lambda \neq$ 0 exists. From

$$
\beta_{1} \lambda=\beta_{1} \lim _{i \in \mathbb{Z}, i \rightarrow \infty} \beta_{1}^{i}=\lim _{i \in \mathbb{Z}, i \rightarrow \infty} \beta_{1}^{i}=\lambda,
$$

we deduce that $\beta_{1}=1$.

We now assume that all $a_{k}$ are not zero and $r>1$. Write complex numbers in polar form: $\beta_{k}=e^{\theta_{k}}, b_{k}=\rho_{k} e^{\omega_{k}}$ for $1 \leq k \leq r$. Then

$$
\lim _{i \in \mathbb{Z}, i \rightarrow \infty}\left(a_{1} \beta_{1}^{i}+a_{2} \beta_{2}^{i}+\ldots+a_{r} \beta_{r}^{i}\right)=\lim _{i \in \mathbb{Z}, i \rightarrow \infty}\left(\sum_{k=1}^{r} \rho_{k} e^{i \theta_{k}+\omega_{k}}\right)=\lambda \in C
$$

exists. For any real number $\theta$ we define $\bar{\theta}$ to be such a real number that $0 \leq$ $\bar{\theta}<2 \pi$ and $\bar{\theta} \equiv \theta(\bmod 2 \pi)$. Since $0 \leq \overline{i \theta_{k}}<2 \pi$ for all $i \in \mathbb{Z}$ and for all $1 \leq k \leq r$, there exists a series of integers $\left\{p_{i}\right\}_{i=1}^{\infty}$ such that $\lim _{i \rightarrow \infty} p_{i}=\infty$ and $\lim _{i \rightarrow \infty} \overline{p_{i} \theta_{1}}=\lambda_{1}$ exists. Similarly there exists a sub-series $\left\{h_{i}\right\}_{i=1}^{\infty}$ of $\left\{p_{i}\right\}_{i=1}^{\infty}$ such that $\lim _{i \rightarrow \infty} \overline{h_{i} \theta_{k}}=\lambda_{k}$ exists for all $1 \leq k \leq r$.

Then for any $j \in \mathbb{Z}$, we have

$$
\lambda=\lim _{i \in \mathbb{Z}, i \rightarrow \infty}\left(\sum_{k=1}^{r} \rho_{k} e^{h_{i} \theta_{k}+j \theta_{k}+\omega_{k}}\right)=\sum_{k=1}^{r} \rho_{k} e^{j \theta_{k}} e^{\lambda_{k}+j \theta_{k}+\omega_{k}}=\sum_{k=1}^{r} \rho_{k} \beta_{k}^{j} e^{\lambda_{k}+\omega_{k}} .
$$

It follows that, for all $j \in \mathbb{Z}$,

$$
\begin{gathered}
\sum_{k=1}^{r} \rho_{k} \beta_{k}^{j} e^{\lambda_{k}+\omega_{k}}=\lambda, \\
\sum_{k=1}^{r} \beta_{k} \rho_{k} \beta_{k}^{j} e^{\lambda_{k}+\omega_{k}}=\lambda, \\
\cdots \cdots \\
\sum_{k=1}^{r} \beta_{k}^{r} \rho_{k} \beta_{k}^{j} e^{\lambda_{k}+\omega_{k}}=\lambda .
\end{gathered}
$$


The coefficient matrix of the above set of linear equations is a Vandermonde matrix which is invertible. Thus $\rho_{k} \beta_{k}^{j} e^{\lambda_{k}+\omega_{k}} \neq 0$ is independent of $j$. Therefore $\beta_{k}=1$ for all $1 \leq k \leq r$, which is a contradiction. This completes the proof of this lemma.

Theorem 2.4. Suppose $q$ is a primitive $m$-th root of unity, $\omega_{1}, \omega_{2}, \ldots, \omega_{m}$ are all the $m$-th roots of unity with $m>1$. Then the module $V(\phi)$ over $\widetilde{C}_{q}$ has finite dimensional weight spaces with respect to $d_{1}$ if and only if there exist a positive integer $r$ and $\alpha_{1}, \ldots, \alpha_{r} \in C^{*}$ whose $m$-th powers are pair-wise distinct, $b_{10}^{(k)}, b_{11}^{(k)}, \ldots, b_{1 s_{1}}^{(k)}, \ldots, b_{r 0}^{(k)}, b_{r 1}^{(k)}, \ldots, b_{r s_{r}}^{(k)} \in C$ for $k: 1 \leq k \leq n$ satisfying

$$
\sum_{k=1}^{m} b_{l j}^{(k)}=0, \quad \forall 1 \leq l \leq r, j \geq 0
$$

such that for $i \in \mathbb{Z} \backslash m \mathbb{Z}$,

$$
\begin{aligned}
\phi\left(t_{2}^{i}\right)= & \sum_{k=1}^{m} \frac{\left(b_{10}^{(k)}+b_{11}^{(k)} i+\ldots+b_{1 s_{1}}^{(k)} i^{s_{1}}\right) \omega_{k}^{i} \alpha_{1}^{i}+\ldots+\left(b_{r 0}^{(k)}+b_{r 1}^{(k)} i+\ldots+b_{r s_{r}}^{(k)} i^{s_{r}}\right) \omega_{k}^{i} \alpha_{r}^{i}}{1-q^{i}}, \\
& \phi\left(c_{1}\right)=0 .
\end{aligned}
$$

Proof. " $\Rightarrow$ ". Note that $\phi\left(c_{1}\right)=0$. Suppose $f_{i}=\phi\left(\left(1-q^{i}\right) t_{2}^{i}\right)$ for $i \in \mathbb{Z}$. From Theorem 2.1 we see that

$$
\sum_{i=0}^{n} a_{i} f_{k+i}=0, \quad \forall k \in \mathbb{Z}
$$

Then by a well-known combinatorial formula, we know that there exist $\alpha_{1}, \ldots, \alpha_{r} \in$ $C^{*}$ whose $\mathrm{m}$-th powers are pair-wise distinct, $b_{10}^{(k)}, b_{11}^{(k)}, \ldots, b_{1 s_{1}}^{(k)}, \ldots, b_{r 0}^{(k)}, b_{r 1}^{(k)}, \ldots, b_{r s_{r}}^{(k)}$ $\in C$ for $k: 1 \leq k \leq n$ such that

$f_{i}=\sum_{k=1}^{m}\left(b_{10}^{(k)}+b_{11}^{(k)} i+\ldots+b_{1 s_{1}}^{(k)} i^{s_{1}}\right) \omega_{k}^{i} \alpha_{1}^{i}+\ldots+\left(b_{r 0}^{(k)}+b_{r 1}^{(k)} i+\ldots+b_{r s_{r}}^{(k)} i^{s_{r}}\right) \omega_{k}^{i} \alpha_{r}^{i}, \quad \forall i \in \mathbb{Z}$.

Replacing $i$ with $m i$ in (2.18) we see that

$\sum_{k=1}^{m}\left(b_{10}^{(k)}+b_{11}^{(k)} m i+\ldots+b_{1 s_{1}}^{(k)}(m i)^{s_{1}}\right) \alpha_{1}^{i}+\ldots+\left(b_{r 0}^{(k)}+b_{r 1}^{(k)} m i+\ldots+b_{r s_{r}}^{(k)}(m i)^{s_{r}}\right) \alpha_{r}^{i}=0$.

We may assume that $\left|\alpha_{1}\right| \geq\left|\alpha_{2}\right| \geq \ldots \geq\left|\alpha_{r}\right|,\left|\alpha_{1}\right|=\left|\alpha_{2}\right|=\ldots=\left|\alpha_{r_{1}}\right|>\left|\alpha_{r_{1}+1}\right|$ and $s_{1} \geq \ldots \geq s_{r_{1}}$ in (2.18). Using the fact $\lim _{i \rightarrow \infty} i^{k} \lambda^{i}=0$ for any $\lambda:|\lambda|<1$, from (2.18) we see that

$$
\begin{gathered}
\lim _{i \rightarrow \infty} \frac{\sum_{k=1}^{m}\left(b_{10}^{(k)}+b_{11}^{(k)} m i+\ldots+b_{1 s_{1}}^{(k)}(m i)^{s_{1}}\right) \alpha_{1}^{i}+\ldots+\left(b_{r 0}^{(k)}+b_{r 1}^{(k)} m i+\ldots+b_{r s_{r}}^{(k)}(m i)^{s_{r}}\right) \alpha_{r}^{i}}{i^{s_{1}}\left|\alpha_{1}\right|^{i}} \\
=\lim _{i \rightarrow \infty} \frac{\sum_{k=1}^{m}\left(b_{1 s_{1}}^{(k)} m^{s_{1}}\right) \alpha_{1}^{i}+\ldots+\sum_{k=1}^{m}\left(b_{r_{1} s_{1}}^{(k)} m^{s_{1}}\right) \alpha_{r_{1}}^{i}}{\left|\alpha_{1}\right|^{i}}=0 .
\end{gathered}
$$


Using Lemma 2.3, we deduce that $\sum_{k=1}^{m} b_{1 s_{1}}^{(k)}=\ldots=\sum_{k=1}^{m} b_{r_{1} s_{1}}^{(k)}=0$. In this manner, by repeatedly doing this, we deduce that $\sum_{k=1}^{m} b_{l j}^{(k)}=0$ for all $1 \leq l \leq$ $r, j \geq 0$.

"६". Let $P\left(t_{2}\right)=\prod_{k=1}^{m} \prod_{i=1}^{r}\left(t_{2}-\omega_{k} \alpha_{i}\right)^{s_{i}+1}$. By using the combinatorial formula we can easily verify that (2.17) is true, i.e., (2.4) holds. This completes the proof of this theorem.

Theorem 2.5. Suppose $q$ is a primitive $m$-th root of unity with $m>1$, $\omega_{1}, \omega_{2}, \ldots, \omega_{m}$ are all the $m$-th roots of unity. Then the module $V(\phi)$ over $\widehat{C}_{q}$ has finite dimensional weight spaces if and only if there exist a positive integer $r$ and $\alpha_{1}, \ldots, \alpha_{r} \in C^{*}$ whose $m$-th powers are pair-wise distinct,

$$
b_{10}^{(k)}, b_{11}^{(k)}, \ldots, b_{1 s_{1}}^{(k)}, \ldots, b_{r 0}^{(k)}, b_{r 1}^{(k)}, \ldots, b_{r s_{r}}^{(k)} \in C
$$

for $k: 1 \leq k \leq m$ such that one of the following holds

(a). $\sum_{k=1}^{m} b_{l j}^{(k)}=0 \forall l \geq 1, j \geq 0$, and for $i \in \mathbb{Z} \backslash m \mathbb{Z}$,

$$
\begin{aligned}
& \phi\left(t_{2}^{i}\right)=\sum_{k=1}^{m} \frac{\left(b_{10}^{(k)}+b_{11}^{(k)} i+\ldots+b_{1 s_{1}}^{(k)} i^{s_{1}}\right) \omega_{k}^{i} \alpha_{1}^{i}+\ldots+\left(b_{r 0}^{(k)}+b_{r 1}^{(k)} i+\ldots+b_{r s_{r}}^{(k)} i^{s_{r}}\right) \omega_{k}^{i} \alpha_{r}^{i}}{1-q^{i}}, \\
& \phi\left(c_{1}\right)=0
\end{aligned}
$$

(b). $\alpha_{1}=1, \sum_{k=1}^{m} b_{l j}^{(k)}=0 \forall l \geq 1, j \geq 1$ such that for $i \in \mathbb{Z} \backslash m \mathbb{Z}$,

$\phi\left(t_{2}^{i}\right)=\sum_{k=1}^{m} \frac{\left(b_{10}^{(k)}+b_{11}^{(k)} i+\ldots+b_{1 s_{1}}^{(k)} i^{s_{1}}\right) \omega_{k}^{i} \alpha_{1}^{i}+\ldots+\left(b_{r 0}^{(k)}+b_{r 1}^{(k)} i+\ldots+b_{r s_{r}}^{(k)} i^{s_{r}}\right) \omega_{k}^{i} \alpha_{r}^{i}}{1-q^{i}}$,

$\phi\left(c_{1}\right)=\sum_{k=1}^{m} b_{10}^{(k)}$

Proof. For $V(\phi)$, if $\phi\left(c_{1}\right)=0$, this theorem follows directly from Theorem 2.4. So we now assume that $\phi\left(c_{1}\right) \neq 0$.

" $\Rightarrow$ ". Suppose $f_{i}=\phi\left(\left(1-q^{i}\right) t_{2}^{i}\right)$ for $i \in \mathbb{Z} \backslash m \mathbb{Z}$ and $f_{l m}=\phi\left(c_{1}\right)$ for $l \in \mathbb{Z}$.

Then (2.5) becomes

$$
\sum_{i=0}^{n} a_{i} f_{k+i}=0, \quad \forall k \in \mathbb{Z} .
$$

By a well-known combinatorial formula, we know that there exist $\alpha_{1}, \ldots, \alpha_{r} \in C^{*}$ whose $\mathrm{m}$-th powers are pair-wise distinct, $b_{10}^{(k)}, b_{11}^{(k)}, \ldots, b_{1 s_{1}}^{(k)}, \ldots, b_{r 0}^{(k)}, b_{r 1}^{(k)}, \ldots, b_{r s_{r}}^{(k)} \in$ $C$ for $k: 1 \leq k \leq n$ such that for $i \in \mathbb{Z}$,

$$
f_{i}=\sum_{k=1}^{m}\left(b_{10}^{(k)}+b_{11}^{(k)} i+\ldots+b_{1 s_{1}}^{(k)} i^{s_{1}}\right) \omega_{k}^{i} \alpha_{1}^{i}+\ldots+\left(b_{r 0}^{(k)}+b_{r 1}^{(k)} i+\ldots+b_{r s_{r}}^{(k)} i^{s_{r}}\right) \omega_{k}^{i} \alpha_{r}^{i} .
$$

We deduce that,

$$
\phi\left(t_{2}^{i}\right)=\sum_{k=1}^{m} \frac{\left(b_{10}^{(k)}+b_{11}^{(k)} i+\ldots+b_{1 s_{1}}^{(k)} i^{s_{1}}\right) \omega_{k}^{i} \alpha_{1}^{i}+\ldots+\left(b_{r 0}^{(k)}+b_{r 1}^{(k)} i+\ldots+b_{r s_{r}}^{(k)} i^{s_{r}}\right) \omega_{k}^{i} \alpha_{r}^{i}}{1-q^{i}},
$$


for all $i \in \mathbb{Z} \backslash m \mathbb{Z}$, and

$$
\begin{aligned}
& \phi\left(c_{1}\right)=\sum_{k=1}^{m}\left(b_{10}^{(k)}+b_{20}^{(k)}+\ldots+b_{r 0}^{(k)}\right) \\
& =\sum_{k=1}^{m}\left[\left(b_{10}^{(k)}+b_{11}^{(k)} m i+\ldots+b_{1 s_{1}}^{(k)}(m i)^{s_{1}}\right) \alpha_{1}^{m i}+\ldots+\left(b_{r 0}^{(k)}+b_{r 1}^{(k)} m i+\ldots+b_{r s_{r}}^{(k)}(m i)^{s_{r}}\right) \alpha_{r}^{m i}\right],
\end{aligned}
$$

for all $i \in \mathbb{Z}$. Since $\phi\left(c_{1}\right) \neq 0$, we see that

$$
\phi\left(c_{1}\right)=\sum_{k=1}^{m}\left(b_{10}^{(k)}+b_{20}^{(k)}+\ldots+b_{r 0}^{(k)}\right)=\lambda \neq 0 .
$$

Then (2.21) becomes

$$
\begin{gathered}
\sum_{k=1}^{m}\left[\left(b_{10}^{(k)}+b_{11}^{(k)} m i+\ldots+b_{1 s_{1}}^{(k)}(m i)^{s_{1}}\right) \alpha_{1}^{m i}+\ldots+\left(b_{r 0}^{(k)}+b_{r 1}^{(k)} m i+\ldots+b_{r s_{r}}^{(k)}(m i)^{s_{r}}\right) \alpha_{r}^{m i}\right] \\
=\lambda \neq 0,
\end{gathered}
$$

for all $i \in \mathbb{Z}$. If all $\left|\alpha_{i}\right|<1$, it is clear that $\lambda=\lim _{i \rightarrow \infty}\left(\sum_{k=1}^{m}\left[\left(b_{10}^{(k)}+b_{11}^{(k)} m i+\right.\right.\right.$ $\left.\left.\left.\ldots+b_{1 s_{1}}^{(k)}(m i)^{s_{1}}\right) \alpha_{1}^{m i}+\ldots+\left(b_{r 0}^{(k)}+b_{r 1}^{(k)} m i+\ldots+b_{r s_{r}}^{(k)}(m i)^{s_{r}}\right) \alpha_{r}^{m i}\right]\right)=0$, which is impossible. Using the similar discussion as used in the proof of Theorem 2.4, we deduce that

$$
\begin{gathered}
\sum_{k=1}^{m} b_{l j}^{(k)}=0, \quad \forall l \geq 1, j \geq 1, \quad \text { and } \\
\sum_{k=1}^{m}\left[b_{10}^{(k)} \alpha_{1}^{m i}+\ldots+b_{r 0}^{(k)} \alpha_{r}^{m i}\right]=\lambda \neq 0, \quad \forall i \in \mathbb{Z} .
\end{gathered}
$$

By using Lemma 2.3, we know that one of $\alpha_{j}$ is 1 , say $\alpha_{1}=1$, and

$$
\sum_{k=1}^{m} b_{l 0}^{(k)}=0, \quad \forall l>1 .
$$

Thus we have proved this direction.

"६". Let $P\left(t_{2}\right)=\prod_{k=1}^{m} \prod_{i=1}^{r}\left(t_{2}-\omega_{k} \alpha_{i}\right)^{s_{i}+1}$. By using the combinatorial formula we can easily verify that $(2.19)$ holds, i.e., (2.5) holds. This completes the proof of this theorem.

Example 1. If $\phi=0$, then $V(\phi)$ is the 1-dimensional trivial module.

Example 2. Let $q$ be generic, $\phi\left(c_{1}\right)=0, \phi\left(t_{2}^{2 i}\right)=0, \phi\left(t_{2}^{2 i+1}\right)=\frac{a^{-i}}{1-q^{2 i+1}}$ for all $i \in \mathbb{Z}$. Then $V(\phi)$ has finite dimensional weight spaces.

Example 3. Let $\phi\left(c_{1}\right)=1, \phi\left(t_{2}^{i}\right)=\left\{\begin{array}{ll}\frac{q^{-i}}{1-q^{i}}, & \text { if } q^{i} \neq 1 \\ 1, & \text { if } q^{i}=1\end{array}\right.$ for all $i \in \mathbb{Z}$. Then the module $V(\phi)$ over $\widehat{C}_{q}$ has finite dimensional weight spaces. This module has 
character formula $[6]$

$$
\operatorname{ch}(V(\phi))=\frac{1}{\prod_{i \in \mathbb{N}}\left(1-z^{-i}\right)} .
$$

Remark. In Theorem 2.5(b) if $r=1$ and $s_{1}=1$, then such $V(\phi)$ are all the integrable highest weight modules over the loop Lie algebra $\frac{\mathbf{C}_{q}}{\left(1-t_{2}^{m}\right) \mathbf{C}_{q}} \simeq \widehat{g l}_{m}$ (see $[8])$.

\section{3. $\mathbb{Z} \times \mathbb{Z}$-graded representations for $\widetilde{C}_{q}$}

Since $\widetilde{C}_{q}$ has a natural $\mathbb{Z} \times \mathbb{Z}$-gradation, it is important to consider $\mathbb{Z} \times \mathbb{Z}$ graded modules with finite dimensional homogeneous subspaces. We shall use a technique in $[8,9]$ to construct some irreducible $\mathbb{Z} \times \mathbb{Z}$-graded modules over $L=\widetilde{C}_{q}$ with finite dimensional weight spaces. Note that, with respect to the powers of $t_{1}$ and $t_{2}, \widetilde{C}_{q}$ has a natural $\mathbb{Z} \times \mathbb{Z}$-gradation, but $\widehat{C}_{q}$ does not have such a $\mathbb{Z} \times \mathbb{Z}$-gradation.

Suppose $V(\phi)$ is a $L$-module constructed in Theorems 2.3 and 2.5. Set

$$
\widehat{V}(\phi)=V(\phi) \otimes C\left[y^{ \pm 1}\right] .
$$

If we define the action of $\widehat{L}$ as follows:

$$
\begin{gathered}
\left(t_{1}^{i} t_{2}^{j}\right)\left(u \otimes y^{k}\right)=\left(\left(t_{1}^{i} t_{2}^{j}\right) u\right) \otimes y^{k+j}, \quad \forall u \in V(\phi) ; i, j, k \in \mathbb{Z}, \\
x\left(u \otimes y^{k}\right)=(x u) \otimes y^{k}, \quad \forall u \in V(\phi),
\end{gathered}
$$

where $x \in C c_{1} \oplus C d_{1}$ for $\widehat{C}_{q}, x \in C c_{1} \oplus C d_{1}$ for $\widetilde{C}_{q}$, then $\widehat{V}(\phi)$ becomes an irreducible $\mathbb{Z} \times \mathbb{Z}$-graded $L$-module with finite dimensional weight spaces.

We would like to conclude this paper by asking the following question: in case the irreducible module $V(\phi)$ has finite dimensional weight spaces, can we have a precise expression for $\operatorname{ch} V(\phi)$ ?

\section{References}

[1] S. Berman, Y. Billig, Irreducible representations for toroidal Lie algebras, J. Algebra, 221 (1999), 188-231.

[2] S. Berman, C. Dong, S. Tan, Representations of a class of lattice type vertex algebras, J. Pure Appl. Algebra, 176 (2002), 27-47.

[3] S. Berman, Y. Gao, Y.S. Krylyuk, Quantum tori and the structure of elliptic quasisimple Lie algebras, J. Funct. Anal., 135 (1996), 339-389.

[4] S. Berman, Y. Gao, S. Tan, A unified view of some vertex operator constructions, Israel J. Math., 134 (2003), 29-60.

[5] S. Berman, J. Szmigielski, Principal realization for the extended affine Lie algebra of type $\mathrm{sl}_{2}$ with coordinates in a simple quantum torus with two generators, Recent developments in quantum affine algebras and related topics (Raleigh, NC, 1998), 39-67, Contemp. Math., 248, Amer. Math. Soc., Providence, RI, 1999.

[6] Y. Billig, K. Zhao, Vertex operator representations of quantum tori at roots of unity, Comm. Contemp. Math., 6, (2004) 195-220.

[7] S. Eswara Rao, R.V. Moody, Vertex representations for $n$-toroidal Lie algebras and a generalization of the Virasoro algebra, Comm. Math. Phys., 159 (1994), 239-264. 
[8] Y. Gao, Representations of extended affine Lie algebras coordinatized by certain quantum tori, Compositio Math., 123 (2000), 1-25.

[9] Y. Gao, Vertex operators arising from the homogeneous realization for $\widehat{\mathrm{gl}}_{N}$, Comm. Math. Phys., 211 (2000), 745-777.

[10] M. Golenishcheva-Kutuzova, D. Lebedev, Vertex operator representation of some quantum tori Lie algebras, Comm. Math. Phys., 148 (1992), 403-416.

[11] J. C. McConnell, J. J. Pettit, Crossed products and multiplicative analogues of Weyl algebras, J. London Math. Soc. 38 (1988), 47-55.

[12] K. Zhao, Weyl type algebras from quantum tori, preprint, pp. 30.

School of Mathematics, Tata Institute of Fundamental Research, Homi Bhabha Road, Mumbai-400005, India.

E-mail address: senapati@math.tifr.res.in

Department of Mathematics, Wilfrid Laurier University, Waterloo, ON, N2L 3C5 CANADA

Institute of Mathematics, Academy of Mathematics and System Sciences, Chinese Academy of Sciences, Beijing 100080, P. R. China.

E-mail address: kzhao@wlu.ca 